\title{
The Importance of Measuring Binocular Contrast Sensitivity in Unilateral Cataract
}

\author{
S. PARDHAN AND J. GILCHRIST \\ Bradford
}

\begin{abstract}
Summary
Monocular and binocular contrast sensitivities were measured in patients with uniocular cataract. The cataractous eye showed a greater monocular loss at higher spatial frequencies compared to lower spatial frequencies. Binocular contrast sensitivity depended on the contrast sensitivity differences between the two eyes. At low spatial frequencies, where the monocular sensitivity difference was minimal, binocular summation was obtained. As the sensitivity difference increased at higher spatial frequencies, the binocular contrast sensitivity decreased steadily until it reached a level below the sensitivity of the cataractous eye, demonstrating binocular inhibition. The clinical implications of binocular inhibition obtained with uniocular cataract are discussed.
\end{abstract}

Various tests are available for assessing the progress of cataract. Psychophysical tests include measurement of visual acuity, ${ }^{1-2}$ contrast sensitivity ${ }^{3-4}$ and glare sensitivity. ${ }^{5-7}$ Objective measures include retro-illumination Scheimpflug slit-image photography ${ }^{8}$ and digital image analysis. ${ }^{9-10}$ A measurement of the anterior chamber depth gives an indication of the thickness of the lens. ${ }^{11}$

Contrast sensitivity has become an essential diagnostic tool for comprehensive assessment of cataract. The contrast sensitivity function cannot be predicted from the visual acuity score which only measures the smallest visual angle (and therefore highest spatial frequency) that can be resolved at high contrast . Visual acuity does not give any indication of the contrast sensitivity at medium and low spatial frequencies which are important for viewing everyday targets. ${ }^{12-14}$ It is therefore possible for patients with equal visual acuities to have different contrast sensitivity functions at low and medium spatial frequencies resulting in different perception of large objects at low contrasts. The contrast sensitivity loss with cataract depends on the type of cataract. Early senile cataracts produce losses at only medium and high spatial frequencies, ${ }^{15-16}$ diabetic and posterior subcapsular cataracts have been shown to demonstrate a loss over the whole spatial frequency range ${ }^{17}$ whilst early cortical and nuclear cataracts show losses at medium and high spatial frequencies only. ${ }^{4}$

So far, contrast sensitivity loss with cataract has been assessed monocularly on the cataractous eye itself. There appears to be no reference to the measurement of binocular sensitivity in patients with unequal cataract densities. It is well known that when two eyes have equal monocular sensitivities, the binocular sensitivity is higher than monocular. This is called binocular summation, the vari-

From: Clipp Research Laboratory, Department of Optometry, University of Bradford, West Yorkshire BD7 1SP, U.K.

Correspondence to: Dr Shahina Pardhan, Department of Otometry, University of Bradford, West Yorkshire BD7 1DP. 
ous aspects of which have been extensively reviewed. ${ }^{18-19}$ In contrast detection, the magnitude of binocular contrast summation remains more or less equal over a wide range of spatial frequencies. ${ }^{20-21}$ Laboratory studies in which unequal monocular contrast sensitivities produced by placing a neutral density filter of $1.00 \mathrm{log}$ units before one eye show that the binocular sensitivity decreases to a level below the monocular, demonstrating binocular inhibition..$^{22-23}$ Binocular inhibition is a contrast detection analogue of a well
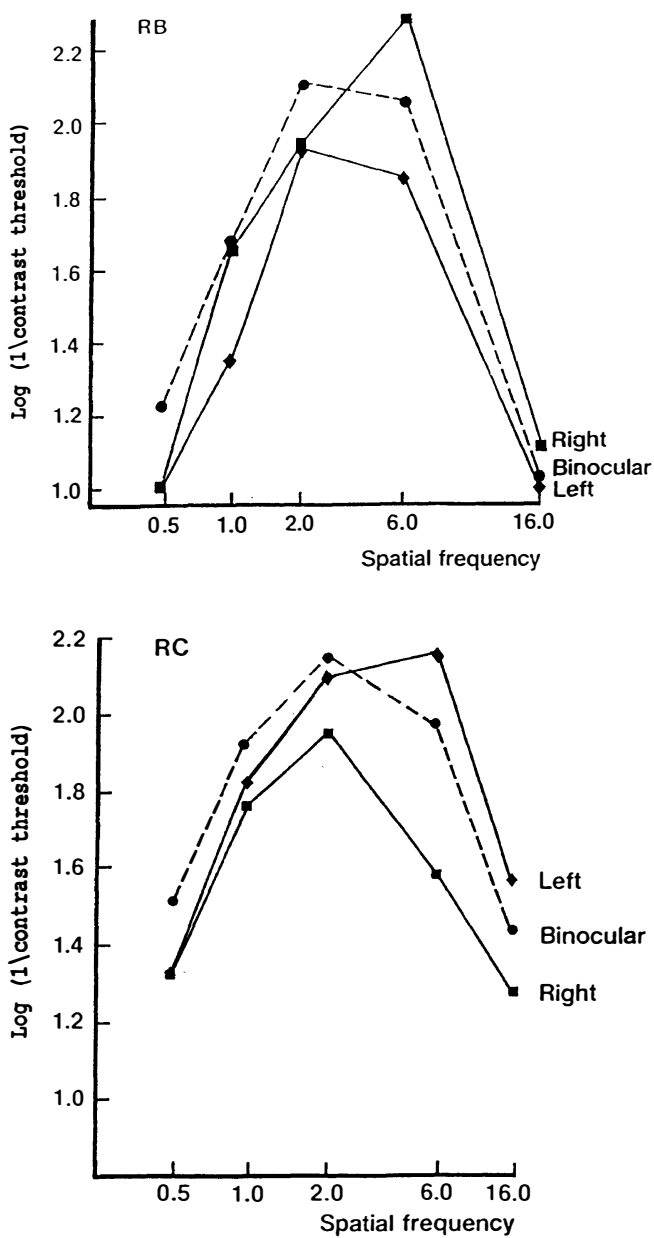

Fig. 1. Contrast sensitivity functions of two of the posterior subcapsular cataract patients. Log (1/contrast threshold) is plotted against spatial frequency. At lower spatial frequencies binocular summation (binocular sensitivity $>$ non-cataractous eye sensitivity) is obtained, while at higher spatial frequencies binocular inhibition (binocular < non-cataractous eye sensitivity) is produced. known phenomenon called Fechner's paradox demonstrated in brightness perception. ${ }^{24-25}$ In the present study, we aim to compare the binocular and monocular contrast functions of patients with uniocular cataract, to investigate the occurrence of binocular summation and inhibition.

\section{Methods}

Eight patients (55-76 years) with unilateral cataract took part in this experiment. Seven patients had early uniocular posterior subcapsular cataract with a visual acuity of $6 / 12$ or better in the cataractous eye. The eighth patient (M.G.) had a long standing unilateral cataract and may have also had a deprivation amblyopia resulting in a visual acuity of $6 / 24$. All patients had a visual acuity of at least $6 / 6$ in their better eye. None of the patients had any visible retinal pathology or intraocular pressures of $21 \mathrm{mmHg}$ (Goldman applanation tonometry). Optimal refractive correction and prismatic corrections were given to each patient for $1 \mathrm{~m}$ viewing distance.

A computerised sinewave generator produced vertical sinusoidal gratings of different spatial frequencies on a Hitachi monitor (phosphor-P4). The circular stimulus field subtended a visual angle of $8^{\circ}$ at a viewing distance of $1 \mathrm{~m}$. The average luminance of the stimulus display was $32 \mathrm{~cd} / \mathrm{m}^{2}$.

To permit a spatial two alternative forced choice (2AFC) procedure, sinewave gratings were generated at random on either the upper or lower half of the screen and the patient indicated on which half the grating appeared. The initial estimate of the threshold was determined by the method of increasing contrast. A thirty trial, two alternative forced choice Quest ${ }^{26}$ algorithm then determined the final threshold estimate at $76 \%$ correct level.

Contrast thresholds were measured for the right eye, left eye and binocularly at five different spatial frequencies $(0.5,1.0,2.0,6.0$ and $16 \mathrm{c} / \mathrm{deg}$ ). The selection for the eye to be tested and the spatial frequency to be measured were randomised.

\section{Results}

The raw data consists of contrast thresholds obtained for the cataractous eye, non-cataractous eye and both eyes, at various spatial 


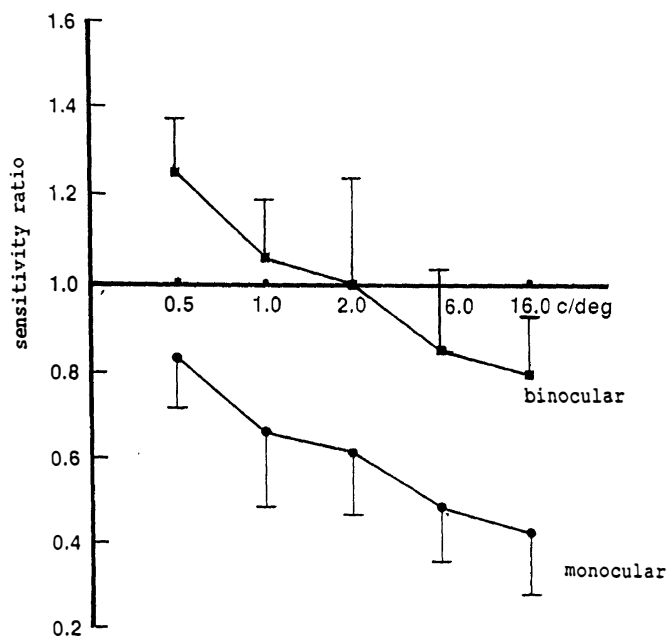

Fig. 2. The average binocular and monocular ratios for the seven posterior subcapsular cataract patients. The binocular ratio is defined as (binocular/noncataract contrast sensitivity), and the monocular sensitivity is defined as (cataract/non-cataract contrast sensitivity). The decrease in contrast sensitivity of the cataractous eye compared to the non-cataractous eye increases as the spatial frequency increases. The binocular sensitivity ratio depends on the difference between the two eyes. When the sensitivity difference between the two eyes is low binocular summation is obtained. As the difference in monocular sensitivities increases at higher spatial frequencies, the binocular sensitivity ratio decreases steadily to reach a level below the monocular, producing binocular inhibition.

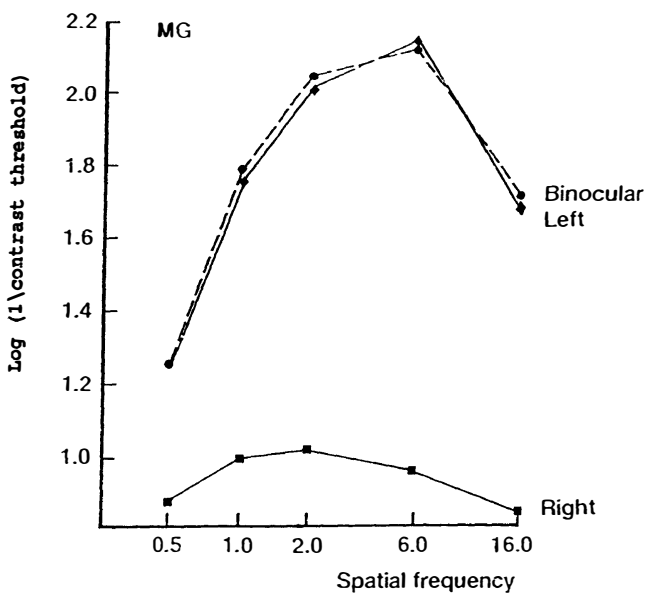

Fig. 3. The contrast sensitivity functions for patient with the dense cataract (M.G.). Log (1/contrast threshold) is plotted against spatial frequency. The binocular contrast sensitivity does not show binocular summation or binocular inhibition. frequencies. Contrast was computed using the Michelson formula (Lmax - Lmin/Lmax + Lmin). Contrast sensitivity is defined as (1/ contrast threshold). Figure 1 shows contrast sensitivity functions where $\log (1 /$ contrast threshold) is plotted against spatial frequency, for two of the posterior subcapsular cataract patients. These two patients represent the results of their group. The cataractous eye shows a lower contrast sensitivity compared to the non-cataractous eye, the difference being greater for higher spatial frequencies than for lower frequencies. Binocular summation, defined as a higher binocular contrast sensitivity compared to the non-cataractous eye, is obtained at lower spatial frequencies. At higher spatial frequencies, the binocular contrast sensitivity decreases to a level below the sensitivity of the non-cataractous eye, showing binocular inhibition. Figure 2 shows the average monocular and binocular sensitivity ratios for the seven posterior subcapsular cataract patients. The monocular ratio defined as (cataract/non-cataract contrast sensitivity), and binocular ratio defined as (binocular/non-cataract contrast sensitivity), is plotted against spatial frequency. A monocular ratio of 1.0 indicates equal contrast sensitivities of the cataractous eye and the non-cataractous eye, while a ratio of less than

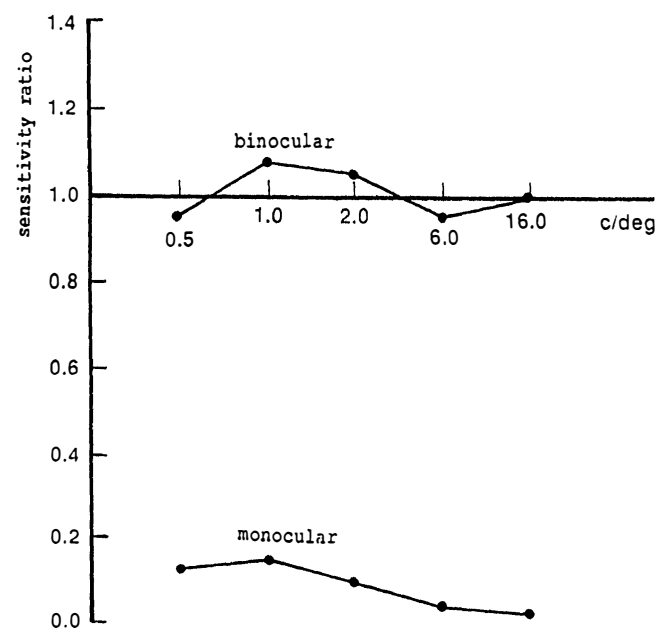

Fig. 4. The binocular and monocular sensitivity ratios for patient M.G. A large difference in contrast sensitivity exists between the two eyes at all spatial frequencies. The binocular sensitivity ratio shows an absence of binocular summation and binocular inhibition, demonstrating suppression of the cataractous eye. 
1.0 shows a lower contrast sensitivity of the cataractous eye compared to the non-cataractous eye. A binocular ratio of greater than 1.0 indicates binocular summation, while a ratio of less than 1.0 shows binocular inhibition. Figure 2 shows that the binocular ratio depends on the difference between the monocular sensitivities. Since it has been shown that equal monocular sensitivities produce binocular summation whose magnitude remains more or less equal at all spatial frequencies, ${ }^{20,21}$ it suggests that binocular inhibition at higher spatial frequencies occurs due to the difference in monocular sensitivities produced by the cataract. At low spatial frequencies where the difference in monocular sensitivities is minimal, binocular summation is obtained. As the difference between the monocular sensitivities increases at higher spatial frequencies, binocular inhibition is produced. The Wilcoxon test showed a significant difference between the binocular and monocular sensitivities at $0.5 \mathrm{c} / \mathrm{deg}$ and 16 $\mathrm{c} / \mathrm{deg}(\mathrm{p}<5 \%)$, indicating definite summation at the low spatial frequency and definite inhibition at the high spatial frequency. Figure 3 shows the contrast sensitivity functions of the long standing dense cataractous patient (M.G.). The binocular and the non-cataractous eye show similar contrast sensitivities at all spatial frequencies, showing no evidence of either binocular summation or inhibition. Figure 4 shows the monocular and binocular sensitivity ratios of the same patient. The monocular ratio shows a large difference between the two eyes and the binocular ratio is more or less equal to unity, at all spatial frequencies. A likely explanation for this is that the dense cataract acts as an occluder producing equal binocular and monocular contrast sensitivities. In such cases, the cataractous eye does not influence the binocular contrast sensitivity.

\section{Discussion}

Binocular inhibition suggests the presence of an interocular inhibitory mechanism between the two eyes. The existence of such inhibitory interactions in interocular masking and binocular rivalry is well known. The origin for these interocular interactions mechanisms may be in the lateral geniculate body $y^{27-28}$ or in the visual cortex. ${ }^{29-32}$
The occurrence of binocular inhibition at low contrast conditions could have important clinical implications. Driving in misty conditions is an example. Uniocular cataractous patients may complain of lower binocular sensitivity compared to the monocular and prefer to shut the cataractous eye. At low and medium spatial frequencies, binocular inhibition would not be revealed by visual acuity. A measurement of the binocular contrast sensitivity at different spatial frequencies would be required to demonstrate binocular inhibition. Another study has shown unequal monocular glare disability also produces binocular inhibition. ${ }^{33}$ It is also well known that cataract increases glare disability. Following this, glare disability in uniocular cataract would affect binocular sensitivity under conditions of glare even though it may not do so under normal conditions.

Cataract extraction is usually performed at the discretion of the ophthalmologist who assesses the cataract using a number of tests including visual acuity and the ability to carry our normal tasks. A uniocular cataract of low density with marginal loss of visual acuity may be left unattended on the basis that the visual acuity of the other eye is normal. However, contrast sensitivity measurement could reveal binocular inhibition, thereby calling for earlier attention. A high correlation between the binocular contrast sensitivity of cataract patients and their perceived visual disability has recently been shown. ${ }^{34} \mathrm{~A}$ realisation that there is a physiological basis for binocular inhibition could be useful in formulating an additional test to assess cataract for surgery.

We would like to thank Dr D. Elliott and Dr W. Douthwaite for useful discussion on this study. Dr Shahina Pardhan is supported by Angelini Pharmaceuticals.

Key words: binocular summation, binocular inhibition, cataract.

\section{References}

${ }^{1}$ Planten JT: Physiologic optic approach of lens and cataracts. Ophthalmologica (Basel) 1975, 171: 249-53.

${ }^{2}$ Brown $\mathrm{N}$ and Hungerford $\mathrm{J}$ : The influence of the size of the lens in ocular disease. Trans Ophthalmol Soc UK 1982, 94: 29-45.

${ }^{3}$ Hess R and Woo G: Vision through cataracts. Invest Ophthalmol Vis Sci 1978, 17: 426-35. 
${ }^{4}$ Elliott DB, Gilchrist J, Whitaker D: Contrast sensitivity and glare sensitivity changes with three types of cataract morphology: are these techniques necessary in a clinical evaluation of cataract? Ophthal Phsyiol Opt 1989, 9: 25-30.

${ }^{5}$ Harbin TS: Visual impairment by sunlight in posterior subcapsular cataract. Ophthal Surg 1973, 4: 34-6.

${ }^{6}$ Paulsson LE and Sjöstrand J: Contrast sensitivity in the presence of a glare light. Invest Ophthalmol Vis Sci 1980, 19: 401-6.

${ }^{7}$ Abrahamsson $\mathbf{M}$ and Sjöstrand $\mathrm{J}$ : Impairment of contrast sensitivity function (CSF) as a measure of disability glare. Invest Ophthalmol Vis Sci 1986, 27: 1131-6.

${ }^{8}$ Brown NP, Bron AJ, Ayliffe W, Sparrow J, Hill AR: The objective assessment of cataract. Eye 1987, 1: 23446.

${ }^{9}$ Brown NP: Quantitative slit-image photography of the lens. Trans Ophthalmol Soc UK 1972, 92: 303-17.

${ }^{10}$ Mayer $\mathrm{H}$ and Irion KM: New approach to area image analysis of Scheimpflug photos of the anterior eye. Ophthal Res 1985, 17: 106-10.

${ }^{11}$ Dermarcelle Y et Luyckx-Bacus J: Biometrie du segment anterieur dans la cataracte senile. Acta Ophthalmol 1971, 49: 454-66.

${ }^{12}$ Owsley C, Sekuler R, Boldt C: Aging and low contrast vision: face perception. Invest Ophthal Vis Sci 1981, 21: 362-5.

${ }^{13}$ Fiorentini A, Maffei L, Sandini G: The role of high spatial frequencies in face perception. Perception 1983, 12: 195-201.

${ }^{14}$ Owsley C and Sloane ME: Contrast sensitivity, acuity, and the perception of real world targets. Br J Ophthalmol 1987, 71: 791-6.

${ }^{15}$ Skalka HW: Arden grating test in evaluating early posterior subcapsular cataracts. South Med J 1981, 74: 1368-70.

${ }^{16}$ Woo G: Contrast sensitivity function as a diagnostic tool in low vision. Am J Optom Physiol Opt 1985 , 62: 648-51.

${ }^{17}$ Howes SC, Caelli T, Mitchell P: Contrast sensitivity in diabetics with retinopathy and cataract. Austral J Ophthalmol 1982, 10: 173-8.

${ }^{18}$ Blake R and Fox R: The psychophysical inquiry into binocular summation. Percept Psychophysics 1973, 14: 161-85.
${ }^{19}$ Blake R, Sloane M, Fox R: Further developments in binocular summation. Percept Psychophysics 1981, 30: 266-76.

${ }^{20}$ Campbell F and Green D: Monocular versus binocular visual acuity. Nature 1985, 208: 191-2.

${ }^{21}$ Pardhan S, Gilchrist J, Douthwaite W: The effect of spatial frequency on binocular contrast inhibition. Ophthal Physiol Opt 1989, 9: 46-9.

${ }^{22}$ Gilchrist J and McIver C: Fechner's paradox in binocular contrast sensitivity. Vis Res 1985, 25: 609-13.

${ }^{23}$ Gilchrist J and Pardhan S: Binocular contrast detection with unequal monocular illuminance. Ophthal Physiol Opt 1987, 7: 373-7.

${ }^{24}$ Engel G: The autocorrelation function and binocular brightness mixing. Vis Res 1969, 9: 1111-30.

${ }^{25}$ Levelt WJ: Binocular brightness averaging and contour information. Br J Psychol 1965, 56: 1-13.

${ }^{26}$ Watson A and Pelli D: QUEST: A Bayesian adaptive psychometric method. Percept Psychophysics 1983, 33: 113-20.

${ }^{27}$ Vastola EF: Monocular inhibition in the lateral geniculate body. EEG Clin Neurophysiol Ophthal 1960, 66: 477-91.

${ }^{28}$ Sanderson KJ, Bishop PO, Darren-Smith I: The properties of binocular receptive fields of lateral geniculate neurones. Exp Brain Res 1971, 13: 178-207.

${ }^{29}$ Poggio G and Fischer B: Binocular interaction and depth sensitivity in striate and prestriate cortex of behaving Rhesus monkey. J Neurophysiol 1977, 40: $1392-405$.

${ }^{30}$ Hubel D and Wiesel T: Receptive fields, binocular interaction and functional architecture in the cat's visual cortex. J Physiol 1962, 160: 106-54.

${ }^{31}$ Henry GH, Bishop PO, Coombs JS: Inhibitory and subliminal excitatory receptive fields of simple units in cat striate cortex. Vis Res 1969, 9: 1289-96.

${ }^{32}$ Abadi RV: Induction Masking-A study of some inhibitory interactions during dichoptic viewing. Vis Res 1976, 16: 269-75.

${ }^{33}$ Pardhan S and Gilchrist J: Binocular contrast sensitivity with monocular glare disability. Ophthal Physiol Opt 1990, 10: 37-9.

${ }^{34}$ Elliott D, Hurst M, Weatherill J: Comparing tests of visual function in cataract with the patient's perceived visual disability. Eye 1990, 4: 712-17. 